\title{
Combined Effect of Nicotine and Caffeine on Orthodontic Tooth Movement in Rats
}

\author{
Naeem Murtaza ${ }^{1}$, Ahmad Shamim $^{2}$, Shabbir Hussain ${ }^{3}$, Muhammad Nauman Sadiq $^{2}$, Muhammad Azeem², $^{2}$ \\ Waheed ul Hamid ${ }^{4}$ \\ ${ }^{1}$ Assistant Professor, Department of Orthodontics, Dental section, Faisalabad Medical University, Faisalabad, Pakistan \\ ${ }^{2}$ Assistant Professor, Department of Orthodontics, De'Montmorency College of Dentistry, Lahore, Pakistan \\ ${ }^{3}$ Assistant Professor, Department of Orthodontics, Rashid Latif Medical \& Dental College, Lahore, Pakistan \\ ${ }^{4}$ Professor/Principal, Department of Orthodontics, De'Montmorency College of Dentistry, Lahore, Pakistan
}

\begin{abstract}
Background: The individual effects of nicotine and caffeine have been reported in previous studies but their combined effect on tooth movement needs to be elucidated. The objective of this study was to evaluate the combined effect of nicotine and caffeine on the magnitude of orthodontic tooth movement (OTM) in rats.

Material and Methods: This experimental study was conducted on Sprague-Dawley rats (Animal House and Pathology Laboratory; Post Graduate Medical Institute, Lahore) in the department of Orthodontics, de'Montmorency College of Dentistry, Lahore from $8^{\text {th }}$ July 2014 to $8^{\text {th }}$ January 2015. Forty male Sprague-Dawley rats were divided into four equal groups: Control group (CR), nicotine group (NT), caffeine group (CF) and combined nicotine and caffeine group (CNC). Closed coil nickel titanium (NiTi) spring was placed between incisor and maxillary molar. Nicotine group (NT) was treated by intraperitoneal injections of nicotine. Caffeine was given to caffeine group and Combined nicotine and caffeine group (CNC) was treated in the same way as individual nicotine and caffeine groups daily for 14 days. All the rats were sacrificed on 15th day. Magnitude of the orthodontic tooth movement was measured using digital Vernier caliper. Means and standard deviation were calculated for orthodontic tooth movement. One-way ANOVA was used to determine the mean difference in OTM. Post hoc Tukey test was used for multiple comparisons among the groups. Results: The mean orthodontic tooth movement (OTM) was $0.32 \mathrm{~mm} \pm 0.05$ in control group, $0.56 \mathrm{~mm} \pm 0.04$ in nicotine group, $0.52 \mathrm{~mm} \pm 0.034$ in caffeine group and $0.8 \mathrm{~mm} \pm 0.06$ in combined NC group, respectively. The difference between mean OTM among the groups was statistically significant $(P$-value $<0.001)$. The mean OTM in CNC group was significantly higher as compared to other groups (CR, NT, CF, NT) $(P$-value $<0.001)$.

Conclusions: In rats, the combined use of nicotine and caffeine results in greater orthodontic tooth movement as compared to their individual use.

Key words: Bone remodeling, Caffeine, Nicotine, Orthodontic tooth movement

Authors' Contribution: Correspondence:

${ }^{1-3}$ Conception; Literature research; Muhammad Azeem

manuscript design and drafting; ${ }^{4-6}$ Critical Email:dental.concepts@hotmail.com

analysis and manuscript review; Data analysis; Manuscript Editing.

Cite this article. Murtaza N, Shamim A, Hussain S, Sadiq MN, Azeem M, Hamid W Combined

Effect of Nicotine and Caffeine on Orthodontic Tooth Movement in Rats. J Islamabad Med

Dental Coll.2020; 9(2): 109-114. Doi: 10.35787/jimdc.v9i2.462

Article info:

Received: November 17, 2019

Accepted: June 14, 2020

Funding Source: Nil

Conflict of Interest: Nil
\end{abstract}




\section{Introduction}

The orthodontic tooth movement (OTM) is based on periodontal ligament (PDL) and alveolar bone remodeling which is a complex process depending upon multiple biological mediators, intercellular and cell to matrix interactions. ${ }^{1}$ The applied mechanical force is converted into molecular events that lead to OTM through multifold networked reactions in alveolar bone and PDL cells. $^{2}$ Any change in the biological response may lead to alteration in bone remodeling process and ultimately OTM. ${ }^{3}$

A number of studies have shown the influence of nicotine on the bone metabolism through its effects on bone remodeling process. ${ }^{4}$ Nicotine stimulated differentiation of osteoclasts in a porcine marrow cell model. ${ }^{5}$ In a rat study, nicotine increased the bone resorption by induction of interleukin -1 , a bone resorbing cytokine. ${ }^{6}$ Similarly a significant increase in the serum levels of bone resorbing cytokines, interleukin-1 (IL-1) and interleukin-6 (IL-6) after administration of nicotine to Sprague-Dawley male rats has also been reported. ${ }^{6,7}$ Investigations on human gingival fibroblasts have shown that nicotine increases the release of prostaglandin E2 (PGE2) by increasing the expression of cyclooxygenase-2 (COX-2) which influence bone resorption. ${ }^{8}$ On the other hand, it is reported that nicotine decreases the production of type 1 collagen and alkaline phosphatase (ALPase) by osteoblasts and suppresses osteogenesis in cultured human osteosarcoma cells. ${ }^{9}$ The use of nicotine resulted in significant acceleration in the rate of orthodontic tooth movement in rats. ${ }^{10}$

Similarly, several studies have demonstrated the effect of caffeine on bone metabolism. Caffeine intake is reported to enhance OTM in rats through its effect on alveolar bone remodeling. ${ }^{11}$ Researchers have found association between reduced bone mineral density and caffeine intake in their experiments on rats. ${ }^{12}$ OTM may be enhanced by daily coffee intake through its effect on bone mineral density. ${ }^{13} \mathrm{~A}$ significant negative relationship between OTM and trabecular bone mass is also demonstrated by Hashimoto et al. in their study on rats. ${ }^{14}$ On the other hand, caffeine is reported to affect the osteoblast function by decreasing 1,25-Dihydroxyvitamin D3 stimulated alkaline phosphatase activity, which is an important marker of osteoblastic activity in human osteoblast cells. ${ }^{15}$

Caffeine is commonly used psychoactive agent by the children and adults because its use is socially acceptable and it is easily available. ${ }^{16}$ Carbonated and energy drinks, chocolates and coffee are rich sources of caffeine. ${ }^{17}$ Similarly, nicotine is found in many plants as naturally occurring alkaloid. Tobacco and other products like nicotine containing gums are the available sources of nicotine. ${ }^{18}$ Out of thousands of drugs that are made in the laboratory or found in nature, caffeine and nicotine are among the most widely used legal drugs worldwide. ${ }^{19}$

Although alterations in OTM through changes in bone remodeling process by the individual effects of nicotine and caffeine have been reported in previous studies but their combined effect on OTM is not reported so far. Many of the young orthodontic patients are simultaneously exposed to nicotine and caffeine thus warranting the need for this study.

The null hypothesis was that combined use of nicotine and caffeine increases the orthodontic tooth movement in a rat model.

\section{Material and Methods}

This experimental study was conducted on male Sprague-Dawley rats, 10-12 weeks old weighing $200 \mathrm{gm}( \pm 20)$ in the department of Orthodontics, 
de'Montmorency College of Dentistry (DCD), Lahore and Pathology Laboratory, Post Graduate Medical Institute, Lahore from $8^{\text {th }}$ July 2014 to $8^{\text {th }}$ January 2015. All steps described herein conform to the ethical principles for animal research adopted by the US National Institutes of Health and were approved by ethical review committee of DCD and Post Graduate Medical Institute, Lahore (Protocol number 4320/19.9.2019).

The sample size of 40 rats was calculated keeping the power of study at $80 \%$ and level of significance to $5 \% .{ }^{10}$ The 40 rats were randomized by lottery method into 4 groups with 10 rats in each group i.e. Control group (CR), Nicotine group (NT), Caffeine group (CF) and Combined Nicotine and Caffeine group (CNC). The rats were housed in the same room and placed in 8 plastic cages (5 per cage), and acclimated to a 12-hour light/dark cycle for 1 week. They had free access to water and standard laboratory rat chow at a temperature of $21^{\circ} \mathrm{C}$ and humidity of $55 \%$. Nicotine group (NT) was treated by intraperitoneal injections of nicotine, $1 \mathrm{mg} / 1000 \mathrm{gm}$ body weight (nicotine tartrate salt, Merck, Darmstadt, Germany, dissolved in normal saline solution). ${ }^{10}$ Caffeine was given to the caffeine group (CF) as $2.5 \mathrm{mg} / 100 \mathrm{gm}$ body weight in drinking water. ${ }^{11}$ Combined nicotine and caffeine group (CNC) was treated in the same way as individual nicotine and caffeine groups daily for 14 days.

Before placement of an orthodontic appliance, rats were sedated with intramuscular injection of 1.8 $\mathrm{mg} / \mathrm{kg}$ ketamine and $1.1 \mathrm{mg} / \mathrm{kg}$ xylazine, and distance between distal surface of right maxillary first molar and mesial surface of right maxillary second molar was measured. This was followed by ligation of orthodontic appliance between incisor and right maxillary first molar by laceback wire (Morelli, São Paulo, SP, Brazil), using light cure composite adhesive (Transbond XT; 3M/Unitek, Monrovia, Calif) after drilling the tooth surface. ${ }^{10}$ This appliance comprised of a nickel titanium (NiTi) closed coil spring (Sentaloy, GAC, NY) delivering a force of $20 \mathrm{cN}$, measured using a dynamometer tension gauge (MedKraft Orthodontics, USA). ${ }^{6,10}$

All the rats were sedated at 15th day after the placement of appliance with the help of $5.4 \mathrm{mg} / \mathrm{kg}$ ketamine to measure the orthodontic tooth movement. This was measured as the distance between distal surface of right maxillary first molar to mesial surface of right maxillary second molar, using calibrated digital Vernier caliper (Fowler High Precision, Newton, Mass). Data was analyzed using SPSS version 20. Means and standard deviation were calculated for orthodontic tooth movement. One-way ANOVA was used to determine the mean difference in OTM. Post-hoc Tukey test was used for multiple comparisons among the groups. A $P$ value $\leq 0.05$ was considered as statistically significant.

\section{Results}

The mean orthodontic tooth movement (OTM) in $\mathrm{CR}, \mathrm{NT}, \mathrm{CF}$ and CNC groups was $0.32 \mathrm{~mm} \pm 0.05$, $0.56 \mathrm{~mm} \pm 0.04,0.52 \mathrm{~mm} \pm 0.034$ and $0.8 \mathrm{~mm} \pm$ 0.06 , respectively. One-way ANOVA was applied to compare the mean OTM among groups, which was found to be statistically significant $(P$-value $<0.001)$ (Figure 1).

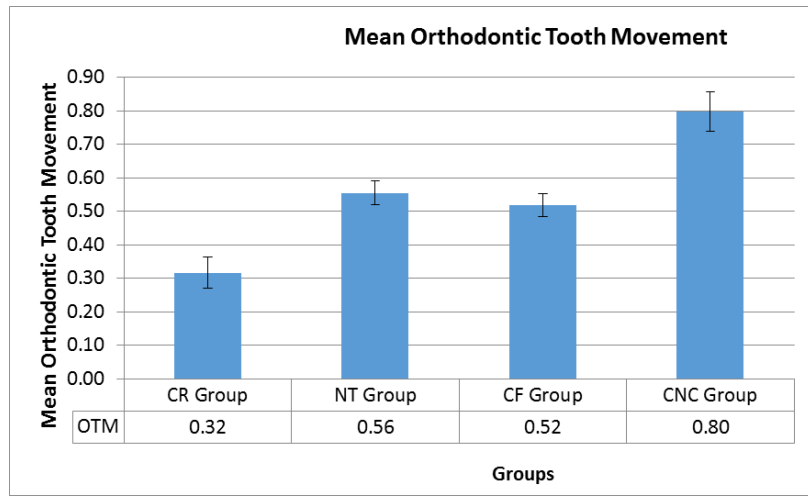

Figure 1: Comparison of mean orthodontic tooth movement among different groups

For multiple comparisons, post hoc Tukey test was used which showed that mean orthodontic tooth 
movement in CNC group was significantly greater as compared to other groups (CR, NT, CN) (Table I).

\begin{tabular}{|c|c|c|c|c|c|}
\hline $\begin{array}{l}\text { S. } \\
\text { No. }\end{array}$ & $\begin{array}{c}\text { Gp } \\
\text { I }\end{array}$ & $\begin{array}{c}\text { Gp } \\
\text { J }\end{array}$ & $\begin{array}{l}\text { MD } \\
(I-J)\end{array}$ & SE & $\begin{array}{c}\text { P- } \\
\text { value* }\end{array}$ \\
\hline \multirow{3}{*}{1} & \multirow{3}{*}{$C R$} & NT & $-.23900^{*}$ & .02005 & $<0.001$ \\
\hline & & $\mathrm{CF}$ & $-.20300^{*}$ & .02005 & $<0.001$ \\
\hline & & CNC & $-.48100 *$ & .02005 & $<0.001$ \\
\hline \multirow{2}{*}{2} & \multirow{2}{*}{ NT } & $\mathrm{CF}$ & 0.03600 & .02005 & 0.292 \\
\hline & & $\mathrm{CNC}$ & $-.24200 *$ & .02005 & $<0.001$ \\
\hline 3 & $\mathrm{CF}$ & $\mathrm{CNC}$ & $-.27800^{*}$ & .02005 & $<0.001$ \\
\hline
\end{tabular}

${ }^{*} P$ value $\leq 0.05$ was considered statistically significant Gp-Group; MD-Mean difference; SE-Standard error

\section{Discussion}

Numerous studies have been conducted in the past in order to monitor the effects of different drugs and surgical procedures on OTM. ${ }^{4-6}$ Some of these studies reported that several agents, drugs and surgical procedures enhanced the OTM, while other studies proved that the process of OTM is slowed down by different stimuli. ${ }^{7,8}$ The combined effects of nicotine and caffeine on OTM need to be investigated. Most of the young smokers exposed to nicotine are simultaneously exposed to caffeine in the form of coffee, chocolates and energy drinks. $^{19}$

We selected nicotine and caffeine the two psychoactive agents which are amongst the most extensively used stimulant drugs in the world. ${ }^{20}$ One of the main reasons for excessive use is that in most of the countries these drugs are legal even if not freely accessible to everyone. ${ }^{19}$ Comparatively there is limited knowledge available about the combined effects of these two agents. ${ }^{21}$

In this study, rats were used as a model for OTM. For the placement of an orthodontic appliance, rats are the preferred choice because mice are too small for an orthodontic appliance to be placed accurately. ${ }^{22}$
At present several methods including elastic power chains, other elastic modules and NiTi springs are being used for application of orthodontic force. However, in case of active modules and elastic chain there is considerable force decay with time which is a potential disadvantage. NiTi springs are considered an alternative, and are used extensively because these springs have advantage of delivering more consistent force for a longer period of time. ${ }^{23}$ For experimental research in the field of orthodontics, application of continuous low force is recommended. ${ }^{22,24} \mathrm{NiTi}$ closed coil spring was also used in this study for delivering continuous orthodontic force of $20 \mathrm{cN}$ measured with a tension gauge. The experimental animals were sacrificed on the 15th day of administration of combined caffeine and nicotine, to measure the OTM. Several phases of OTM have been established by the past clinical and animal research. ${ }^{25}$ In this study the difference in the mean orthodontic tooth movement in all groups was statistically significant $(P<0.001)$, with greater orthodontic tooth movement noted in CNC group and least in CR group in comparison to other groups.

The variation in the underlying biological process is accountable for the difference in amount of OTM. ${ }^{15}$ Henemyre et al. investigated the effect of nicotine on calcium and phosphate resorption of porcine osteoclasts and concluded that nicotine enhanced the resorptive function as well as the number of osteoclasts. ${ }^{5}$ This effect of nicotine may have contributed to an increase in OTM. Rapuri et al. reported that caffeine dose dependently decreased the expression of vitamin $D$ receptors and the activity of enzyme alkaline phosphatase in human osteoblasts. ${ }^{15}$ This explains the possible mechanism by which bone metabolism is affected by caffeine intake. In our study, the greatest amount of OTM was found in CNC group, which was treated with both nicotine and caffeine. However, combined effects of nicotine and caffeine on amount of OTM did not turn out as the summative effect of 
individual drugs. These two drugs may have variable physiological effects when used in combination. Clinical and Epidemiological studies suggest a potential interaction between caffeine and nicotine. ${ }^{26-28}$

It has long been observed by the orthodontists that response to orthodontic treatment is variable in different individuals in terms of rate of tooth movement. This difference in rate of tooth movement is due to the change in the process of bone remodeling which is induced by the various drugs and other systemic factors.

The implication of this study is that a thorough medical history is imperative as a large fraction of young and adult population is exposed to these agents. Consequently, it is essential that the orthodontist should pay attention to the social history of every patient to plan the best strategy for treatment including appointment intervals, force level control and duration of treatment for each class. The findings of this study establish that for increased amount of orthodontic tooth movement, the combination of nicotine and caffeine are recommended. The positive effect of combined nicotine and caffeine is an increase in the rate of orthodontic tooth movement, but a negative effect is that it may cause harm to underlying alveolar bone and orthodontic stability in presence of certain medical conditions. ${ }^{18,19}$

There are certain limitations of this study. It is an experimental animal study with a limited sample size. Findings of an animal study have to be validated through human studies. A limited sample size of animals is an ethical requirement of animal research, which allows researchers to use a minimum number of animals in an experiment. Moreover, effect on bone resorption was not investigated.

Our recommendation is that human studies with larger sample size should be conducted to find out the effect of these agents on orthodontic tooth movement and bone resorption.

\section{Conclusion}

In this animal study, the combined use of nicotine and caffeine resulted in greater orthodontic tooth movement as compared to their individual use. It is recommended that human studies with larger sample size should be conducted to further find out the effect of these agents on orthodontic tooth movement.

\section{References}

1. Meikle MC. The tissue, cellular, and molecular regulation of orthodontic tooth movement: 100 years after Carl Sandstedt. Eur J Orthod. 2006; 28(3): 221-40. Doi: 10.1093/ejo/cjl001

2. Masella RS, Meister M. Current concepts in the biology of orthodontic tooth movement. Am J Orthod Dentofacial Orthop. 2006; 129(4): 458-68. Doi: 10.1016/j.ajodo.2005.12.013

3. Diravidamani K, Sivalingam SK, Agarwal V. Drugs influencing orthodontic tooth movement: An overall review. J Pharm Bioallied Sc. 2012; 4(Suppl 2): S299. Doi: 10.4103/0975-7406.100278

4. Hermizi H, Faizah O, Ima-Nirwana S, Ahmad Nazrun $S$, Luke DA, Norazlina M. Nicotine impaired bone histomorphometric parameters and bone remodeling biomarkers in Sprague-Dawley male rats. Ann Microsc. 2007; 7: 10-24.

5. Henemyre CL, Scales DK, Hokett SD, Cuenin MF, Peacock ME, Parker MH, et al. Nicotine stimulates osteoclast resorption in a porcine marrow cell model. J Periodontol. 2003; 74(10): 1440-6. Doi: 10.1902/jop.2003.74.10.1440

6. Nozrazlina M, Hapidin H, Othman F, Norliza M, Shuid AN, Soelaiman IN. Vitamin $E$ reversed nicotine-induced toxic effects on bone biochemical markers in male rats. Arch Med Sci. 2010; 6(4): 50512. Doi: $10.5114 /$ aoms.2010.14460

7. Hapidin H, Othman F, Soelaiman IN, Shuid AN, Luke DA, Mohamed N. Negative effects of nicotine on bone-resorbing cytokines and bone histomorphometric parameters in male rats. J Bone 
Miner Metab. 2007; 25(2): 93-8. Doi: 10.1007/s 00774-006-0733-9

8. Nakao $S$, Ogata $Y$, Sugiya $H$. Nicotine stimulates the expression of cyclooxygenase-2 mRNA via NFKB activation in human gingival fibroblasts. Arch Oral Bio. 2009; 54(3): 251-7. Doi: 10.1016/j.archoral bio.2008.11.006

9. Tanaka $H$, Tanabe $N$, Suzuki $N$, Shoji $M$, Torigoe $H$, Sugaya $A$, Motohashi $M$, et al. Nicotine affects mineralized nodule formation by the human osteosarcoma cell line Saos-2. Life Sci. 2005; 77(18): 2273-84. Doi: 10.1016/j.Ifs.2005.02.022

10. Sodagar A, Donyavi Z, Arab S, Kharrazifard MJ. Effect of nicotine on orthodontic tooth movement in rats. Am J Orthod Dentofacial Orthop. 2011; 139(3): e261-5. Doi: 10.1016/j.ajodo.2010.08.018

11. Peng S, Chun HY. Effect of caffeine on alveolar bone remodeling during orthodontic tooth movement in rats. J Tongji Med Univ. 2011;3.

12. Lacerda SA, Matuoka RI, Macedo RM, Petenusci SO, Campos AA, Brentegani LG. Bone quality associated with daily intake of coffee: a biochemical, radiographic and histometric study. Braz Dent J. 2010; 21(3): 199-204. Doi: 10.1590/s0103-6440201 0000300004

13. Yi J, Zhang L, Yan B, Yang L, Li Y, Zhao Z. Drinking coffee may help accelerate orthodontic tooth movement. Dent Hypotheses. 2012; 3(2): 72. Doi: 10.4103/2155-8213.100391

14. Hashimoto M, Hotokezaka H, Sirisoontorn I, Nakano $T$, Arita $K$, Tanaka $M$, et al. The effect of bone morphometric changes on orthodontic tooth movement in an osteoporotic animal model. Angle Orthod. 2013; 83(5): 766-73. Doi: 10.2319/111312869.1

15. Rapuri PB, Gallagher JC, Nawaz Z. Caffeine decreases vitamin $D$ receptor protein expression and 1,25 (OH) 2D3 stimulated alkaline phosphatase activity in human osteoblast cells. J Steroid Biochem Mol Biol. 2007; 103(3-5): 368-71. Doi: 10.1016/j.jsbmb. 2006.12.037

16. Temple JL. Caffeine use in children: what we know, what we have left to learn, and why we should worry. Neurosci Biobehav Rev. 2009; 33(6): 793-806. Doi: 10.1016/j.neubiorev.2009.01.001
17. Babu KM, Church RJ, Lewander W. Energy drinks: the new eye-opener for adolescents. Clin Ped Emerg. 2008; 9(1): 35-42. Doi: 10.1016/j.cpem. 2007.12.002

18. Yildiz D. Nicotine, its metabolism and an overview of its biological effects. Toxicon. 2004; 43(6): 619-32. Doi: 10.1016/j.toxicon.2004.01.017

19. Jerome H, KoobGF. Substance abuse and addiction. Dana Guide. 2007.

20. Crocq MA. Alcohol, nicotine, caffeine, and mental disorders. Dialogues Clin Neurosci. 2003; 5(2): 175. PMID: 22033899

21. Johnson MW, Strain EC, Griffiths RR. Effects of oral caffeine pretreatment on response to intravenous nicotine and cocaine. Exp Clin Psychopharmacol. 2010; 18(4): 305. Doi: 10.1037/a0020509

22. Ren $Y$, Maltha JC, Kuijpers-Jagtman AM. The rat as a model for orthodontic tooth movement-a critical review and a proposed solution. Eur J Ortho. 2004; 26(5): 483-90. Doi: 10.1093/ejo/26.5.483.

23. Dixon V, Read MJ, O'brien KD, Worthington HV, Mandall NA. A randomized clinical trial to compare three methods of orthodontic space closure. J Orthod. 2002; 29(1): 31-6. Doi: 10.1093/ortho/ 29.1.31

24. Van Leeuwen EJ, Maltha JC, Kuijpers-Jagtman AM. Tooth movement with light continuous and discontinuous forces in beagle dogs. Eur J Oral Sci. 1999; 107(6): 468-74. Doi: 10.1046/j.0909-8836. 1999.eos107608.x

25. Proffit WR, Fields HW, Sarver DM. Contemporary Orthodontics, Mosby, St. Louis, Mo, USA. 2000.

26. Jones $H E$, Griffiths RR. Oral caffeine maintenance potentiates the reinforcing and stimulant subjective effects of intravenous nicotine in cigarette smokers. Psychopharmaco. 2003; 165(3): 280-90. Doi: 10.100 7/s00213-002-1262-4

27. Gilbert DG, Dibb WD, Plath LC, Hiyane SG. Effects of nicotine and caffeine, separately and in combination, on EEG topography, mood, heart rate, cortisol, and vigilance. Psychophysio. 2000; 37(5): 583-95.

28. Blank MD, Kleykamp BA, Jennings JM, Eissenberg $T$. Caffeine's influence on nicotine's effects in nonsmokers. Am J Health Behav. 2007; 31(5): 47383. Doi: 10.5555/ajhb.2007.31.5.473 